\title{
A noncontact sensor for measurement of distal residual-limb position during walking
}

\author{
Joan E. Sanders, PhD; ${ }^{*}$ Ari Karchin, MSE; John R. Fergason, CPO; Elizabeth A. Sorenson, MSE \\ Departments of Bioengineering and Rehabilitation Medicine, University of Washington, Seattle, WA
}

\begin{abstract}
A simple noncontact device was implemented for measuring the position of the distal residual limb relative to the prosthetic socket during ambulation. The device was a small and lightweight photoelectric sensor positioned within a frame mounted immediately beneath the socket. Calibration tests showed that the sensor had a displacement range of $60.0 \mathrm{~mm}$. The root-mean-square error for all sources of error considered (different reflective surfaces, peak-to-peak signal noise, drift, nonlinearity, different surface tilt angles, surface curvature, and wetness [simulating sweating]) was $<1.95 \%$ full-scale output. We used the sensor in a preliminary study on a unilateral, transtibial amputee with diabetes to assess pistoning during ambulation. Results showed an average $41.7 \mathrm{~mm}$ proximal displacement during swing phase relative to stance phase. When the subject was walking on a flat surface, pistoning was significantly less $(p=0.000)$ with a supracondylar strap compared with no supracondylar strap, although the difference was not substantial $(0.8 \mathrm{~mm})$. A $5 \mathrm{~min}$ rest period caused the limb to displace proximally in the socket approximately $4.8 \mathrm{~mm}$ during subsequent walking trials, possibly reflecting limb enlargement and thus a more proximal position in the socket after the rest period. The device can potentially be used in prosthetics research for evaluating clinical features that may affect limb position and pistoning and thus fit.
\end{abstract}

Key words: ambulation, displacement, interface, liner, pistoning, position, prosthetics, rehabilitation, sensor, socket, transtibial amputee.

\section{INTRODUCTION}

Measurement of the position of the residual limb in the prosthetic socket during walking is potentially useful in both prosthetics research and clinical practice. The amount of slip, or pistoning, between a limb and socket with different suspension systems for different activities could be quantitatively evaluated. Comfortable prosthetic fitting has been correlated with decreased vertical tibial translation [1]. In a clinical setting, when excessive pistoning is deemed detrimental to the patient, a prosthetist could use the measurement to quantify the degree of pistoning and work to minimize the motion.

A number of studies have used traditional imaging methods to assess the positions of bony structures within the residual limb relative to the socket wall. Radiological methods include roentgenology [2-3], cineradiography [1,4-5], fluoroscopy [6], and roentgen stereophotogrammetric analysis [7]. These studies have shown that for transtibial amputee subjects, the vertical motion between the bones and the socket ranged to approximately $40 \mathrm{~mm}$ between the midstance and swing phases. Ultrasonic methods have also been used, in which ultrasound transducers are attached to the socket wall and their subsequent video recordings are analyzed [8-9].

While the bone-to-socket displacement data collected with these techniques provide interesting insight, the methods are not generally available to many researchers or prosthetists because of the cost of the equipment and the

\footnotetext{
Abbreviations: FSO = full-scale output, LVDT = linear variable differential transformer, RMS = root-mean-square.

*Address all correspondence to Joan E. Sanders, PhD; Bioengineering 355061, Foege N430J, 1705 NE Pacific St, University of Washington, Seattle, WA 98195; 206-221-5872; fax: 206-685-3300. Email: jsanders@u.washington.edu
}

DOI: 10.1682/JRRD.2004.11.0143 
lack of portability. In addition, data collection and analysis can be complex and time-consuming. If radiological methods are to be used and the analysis is to occur frequently, then radiation exposure to the patient may be a concern.

In this research, we implemented an easy-to-use nonradiological means to measure the position of the distal residual-limb surface relative to the distal socket during ambulation. Such a device has the potential to provide insight into the dependence of limb position and vertical motion on a range of componentry, treatments, or other features of clinical relevance. It could be used to characterize the pistoning induced by different suspension systems or different limb fluid control treatments for different activities. Clinically, the device could be a useful tool for measuring the amount of pistoning that feels optimal to a patient, information that would be useful during refitting.

\section{METHODS}

A number of devices measure displacement between two surfaces, using capacitive, inductive, reluctive, potentiometric, and strain-gage-based methods [10]. However, all these techniques require the sensor to contact both objects of interest. In this application, a noncontact method is preferred so that the sensor does not distort the residual-limb soft tissues or alter the measurement of interest. Ultrasonic distance measurement devices are possible. However, the smallest commercially available device (UT212, Nivelco, Budapest, Hungary) was too wide and too long to fit within a prosthesis without appreciably extending the limb length. Measurement with this device could have been accomplished with a $90^{\circ}$ reflective mirror positioned within the distal socket in addition to a sensing apparatus extending out laterally. However, the mirror would have been difficult to position and align.

Photoelectric position-sensing is a viable means for this measurement. Although typically used for proximity sensing, photoelectric sensors can be configured to measure absolute position. A photoelectric sensor is made up of a light source and a light sensor. In this research, we used a diffuse reflective sensor, which means that the light source and light sensor are mounted within a common housing and then placed on the same side of an object whose distance from the sensor is to be assessed. A through-beam sensor has the source and sensor on opposite sides of the object and thus would be inappropriate here. With a diffuse reflective sensor, the light hitting the object is scattered diffusely off the surface. The light sensor measures the intensity of the reflected light energy. The output of the photoelectric sensor decreases with distance to the measured object [10]. The measurement is not sensitive to incident angle. By using a light-emitting diode as the light source, one can minimize undesirable noise from ambient light [10].

The photoelectric sensor element we selected (Figure 1) was $6 \mathrm{~mm}$ in diameter, $12 \mathrm{~mm}$ long, and $6.6 \mathrm{~g}$ in mass (BMOA 06TM-X63-R1, Balluff, Inc, Florence, Kentucky). Signal conditioning and amplification were provided within a $52 \times 36 \times 15 \mathrm{~mm}, 60 \mathrm{~g}$ console (BMO A01-H-V1-C-02, Balluff, Inc) that came with the unit and that was connected to the sensor via a $1.4 \mathrm{~mm}$ diameter polyvinyl chloride cable. The sensor/amplifier system had a response time of $1 \mathrm{~ms}$ and an output of 0 to $2.5 \mathrm{~V}$. Thus, the dynamic response was sufficient to easily handle a signal with frequency content between 0 and $60 \mathrm{~Hz}$.

To minimize the limb-length increase introduced by positioning the sensor within the prosthesis, we constructed a custom support frame. Since the sensor was of small diameter, most of the length could fit within a $25 \mathrm{~mm}$ diameter hole made in the distal socket and the support frame. The frame held the sensor vertically (Figure 2). The signal conditioner was mounted to the lateral socket wall.

To allow adjustment of the working range of the sensor in the event the distal residual limb was very close to or very far from the distal socket surface, we designed a vertical position adjustment apparatus that was incorporated into the frame. This unit allowed the vertical position of the sensor to be adjusted without doffing the prosthesis. The position was adjusted so that the sensor-to-distal

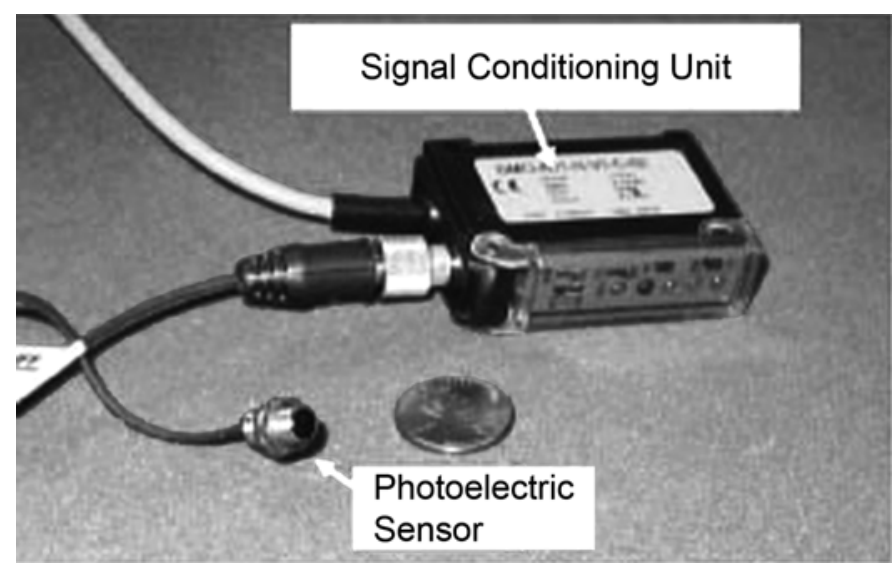

Figure 1.

Photoelectric sensor (6 mm diameter, $12 \mathrm{~mm}$ long) (Balluff Inc, Florence, Kentucky) and signal conditioning unit used in this research. 


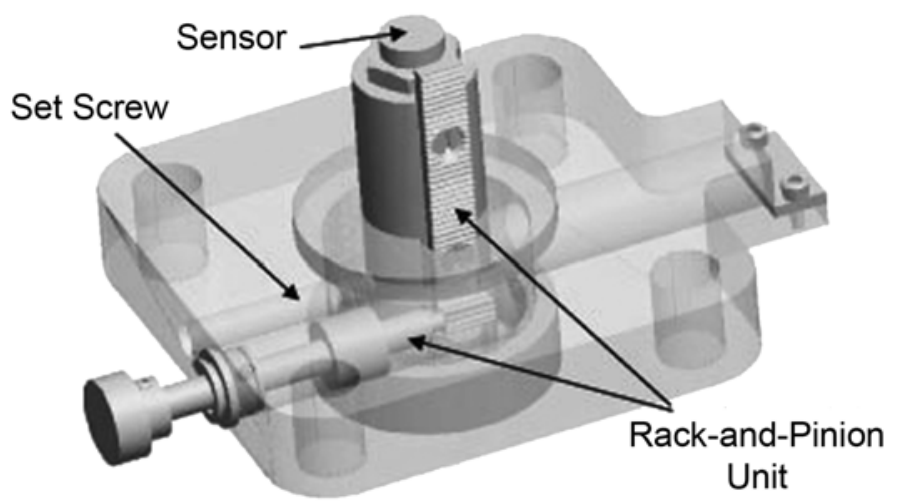

Figure 2.

Perspective view of rack-and-pinion unit (within frame that holds sensor). Rack and pinion allowed vertical adjustment of sensor via knob at lower left. Set screw locked position.

residual-limb position was within the working range of the sensor. The adjustment unit was a rack and pinion, with the knob positioned laterally so that it was easily accessible (Figure 2). A set screw was tightened to lock the sensor in place once the position was set. The addition of the rack and pinion unit extended the total length of the device. However, because in our application we positioned a custom load cell (similar to that in Berme [11]) that had a $25 \mathrm{~mm}$-diameter hole down its axis beneath the limb position sensor and because the rack and pinion unit could fit within this space, the total length of the prosthesis did not need to be extended the length of the rack and pinion. Since this space was completely enclosed, air from within the socket could not escape, and thus no loss of suspension occurred. The total mass of the sensor and frame was $70 \mathrm{~g}$; it added $8.9 \mathrm{~mm}$ (the thickness of the frame) to the socket length.

We calibrated the sensor using an amputee residuallimb sock as the reflective surface. A $60 \times 40 \mathrm{~mm}$ sample from a nylon residual-limb sock (1.4 mm thickness) was cut and held at its edges in clamps to a flat plate. The plate was fastened to the arm of a digital height gauge (HDS$8 \mathrm{M}$, Mitutoyo, Japan) so that it moved the distance recorded by the calipers. The plate was fastened with a locking nut such that the angle of the plate relative to the vertical axis of the height gauge could be adjusted and locked. The height gauge, accurate to $0.01 \mathrm{~mm}$, was the calibration standard.

We conducted the calibration in at least 10 steps within the working range of the transducer, in first the increasing and then the decreasing distance direction. The output from the sensor signal conditioner was connected to a computer- based custom data acquisition system used to measure interface stresses on lower-limb amputees [12]. This was a 45-channel data acquisition system, of which we used 44 channels to collect interface stress or shank load data and 1 channel for the distal-limb sensor. Signals from the transducers and sensor were sent to signal-conditioning boards within a fanny pack $(1.8 \mathrm{~kg})$ carried by the subject. For the distal-limb sensor, we used a gain of 1 . The 45 signals were multiplexed onto three channels and then sent to an analog-to-digital board (MIO-16XE-10, National Instruments Corp, Austin, Texas) within a personal computer (450 MHz, Optiplex, Dell, Inc, Round Rock, Texas). We used a custom Labview program (National Instruments Corp.) to control data acquisition. Data were acquired at a $175 \mathrm{~Hz}$ sampling rate from each channel for a total trial time interval specified by the user. During calibration testing, we collected data at each calibration step for a 2 s interval and calculated the mean voltage. We used a $2 \mathrm{~s}$ interval to achieve a reasonable temporal average for each calibration point. We used a third-order polynomial to fit the voltage versus distance data. The sensor was recalibrated after the human subject studies described later in this section.

We conducted evaluation tests to assess the influence of reflective surface material, incident angle, surface curvature, and fluid presence (simulating sweating) on the measurement. Materials tested were leather (1.4 mm thickness, to simulate skin), a nylon residual-limb sock $(1.4 \mathrm{~mm}$ thickness), and a wool residual-limb sock (3.0 mm thickness). We used a flat plate covered with the leather, nylon sock, or wool sock to assess the dependence of the measurement on incident angle. Angles of the surface relative to the horizontal ranging to $30^{\circ}$ were evaluated. We selected this range because we expected it to be typical of that in the central region of the distal residual-limb surface, based on previously collected residual-limb shape data [13]. A curved surface (hemisphere shape, $38 \mathrm{~mm}$ diameter) made from an inflated balloon positioned within a nylon sock was also tested. We used this dimension because we believed it represented a small-sized residual limb and thus a high but acceptable curvature condition. We assessed fluid presence by adding approximately $1 \mathrm{~mL}$ of water to the surface of the sock material mounted to the flat plate and then conducting a calibration test. Water was added to the surface closest to the sensor.

We conducted additional evaluation tests to assess error in the system. Peak-to-peak noise was defined as the maximum voltage minus the minimum voltage over a $2 \mathrm{~s}$ sampling interval with the sensor held at a constant position. We evaluated drift over a $60 \mathrm{~min}$ interval by sampling at 
a $0.017 \mathrm{~Hz}$ rate with the sensor held at a constant position. We chose a $60 \mathrm{~min}$ interval because we believed it represented the duration of a data collection session on an amputee subject. The drift error was defined as the maximum voltage minus the minimum voltage over the 60 min interval. We evaluated dynamic response by comparing the sensor output with that from a linear variable differential transformer (LVDT) (200-DCD, Schaevitz, Pennsauken, New Jersey). According to manufacturer specifications, the LVDT had a $-3 \mathrm{~dB}$ point at $250 \mathrm{~Hz}$. The sensor and LVDT were mounted in a test jig such that they both measured the position of a flat, horizontally mounted, aluminum cantilever. We applied loads at a frequency up to $60 \mathrm{~Hz}$ to the cantilever, sampled the output from the devices using a $60 \mathrm{MHz}$ storage oscilloscope, and assessed the response differences between the sensor and the LVDT. We used a 0 to $60 \mathrm{~Hz}$ range because this covered the maximum bandwidth expected based on related data on transtibial amputee interface stress [14]. We used an oscilloscope instead of the computer data acquisition system to ensure an adequate sampling rate for this dynamic assessment.

As a preliminary effort to evaluate the sensor in prosthetics practice, we mounted the sensor within a test prosthesis for a unilateral transtibial male amputee subject who was 60 years of age, $1.8 \mathrm{~m}$ tall, and $100 \mathrm{~kg}$ mass. We obtained internal review board approval and informed consent before conducting these tests. The subject had undergone a transtibial amputation as a result of traumatic injury 6 years prior. For the last 5 years he had diabetes. The test prosthesis was a patellar-tendon-bearing socket with a twoply wool sock and no liner, an endoskeletal pyramid alignment system, a $30 \mathrm{~mm}$ aluminum pylon, a Seattle LightFoot $^{\mathrm{TM}}$ (Seattle Systems, Poulsbo, Washington), and a neoprene sleeve. In some trials, we used a circumferential strap to secure the neoprene sleeve proximal to the femoral condyles, referred to later in this article as a supracondylar strap. The total limb mass was $2.49 \mathrm{~kg}$. The distal-limbposition sensor and load cell were positioned between the socket and alignment jig. The test prosthesis was also instrumented with 13 interface stress transducers [12], although we did not include data from these transducers in the analysis of this study since our intent was only to evaluate the distal-limb-position sensor. The sensors and mounts, $320 \mathrm{~g}$ in total mass and $6.35 \mathrm{~mm}$-diameter surfaces were positioned at relatively flat locations, thus presence of the transducers did not appreciably alter the inside socket shape or weigh down the prosthesis. Data were collected at a 175 $\mathrm{Hz}$ sampling rate for $30 \mathrm{~s}$ intervals while the subject walked the length of an $18.5 \mathrm{~m}$ long hallway with a vinyl floor. The subject's walking speed was monitored during testing [15] and kept to within \pm 7 percent of his normal walking speed of $1.1 \mathrm{~m} / \mathrm{s}$. We conducted three trials, then removed the supracondylar strap and conducted three more trials. Then we allowed the subject a rest period of approximately 5 min, during which he stood still and bore his weight on the contralateral limb. The prosthetic limb contacted the floor with minimal weight-bearing. We then conducted three additional walking trials.

We converted voltage from the sensor to position (in millimeters) using the calibration results. We analyzed data from only the central 11 strides (of 14) from each trial to eliminate strides when the subject was accelerating or decelerating. We determined the maximum and minimum vertical position per stride. Then we calculated an average maximum and minimum position across the strides for each test configuration, and determined an average difference. We used student $t$-tests with a test statistic of 0.05 to compare groups.

\section{RESULTS}

Calibration results showed that the sensor measured distance effectively over a $60.0 \mathrm{~mm}$ range, between 40.0 and $100.0 \mathrm{~mm}$ from the sensor surface. No significant hysteresis was shown in the calibration results. The sensitivity ranged from $0.17 \mathrm{~V} / \mathrm{mm}$ at short distances to $0.01 \mathrm{~V} / \mathrm{mm}$ at long distances. The nonlinear curve fit well to a third-order polynomial, which was used because it produced the best fit of those considered (linear, up to a fourth-order polynomial, exponential, power). The root-mean-square (RMS) error between the experimental data and the curve fit data was 0.03 percent of the full-scale output (FSO). The peakto-peak noise during the calibration tests (maximum minus minimum voltage during each $2 \mathrm{~s}$ test) averaged 0.45 percent FSO. The drift measured over a $60 \mathrm{~min}$ time span was 0.08 percent FSO. Thus the total RMS error in the calibration measurement was 0.15 percent FSO. No significant difference existed in calibration results after the sensor was used in human subject studies.

Evaluation tests showed little sensitivity to reflective material among the three materials tested-leather, nylon, and wool. We made a third-order polynomial fit to all test data from the three materials (Figure 3) and then assessed the RMS error for each material. The errors were $0.42,0.38$, and 0.69 percent FSO for leather, nylon, and wool, respectively. 
Tilt of the reflective surface affected performance (Figure 4). At a $15^{\circ}$ tilt angle, errors were 1.01, 1.08, and 1.33 percent FSO for leather, nylon, and wool, respectively. At a $30^{\circ}$ tilt, errors increased to $1.23,1.61$, and 1.69 percent FSO, respectively. A spherical surface $38 \mathrm{~mm}$ in diameter (balloon inside a nylon sock) introduced an error of 1.64 percent FSO.

Wetness, simulating sweat, had some effect on performance (Figure 5). If a wet wool material was used,

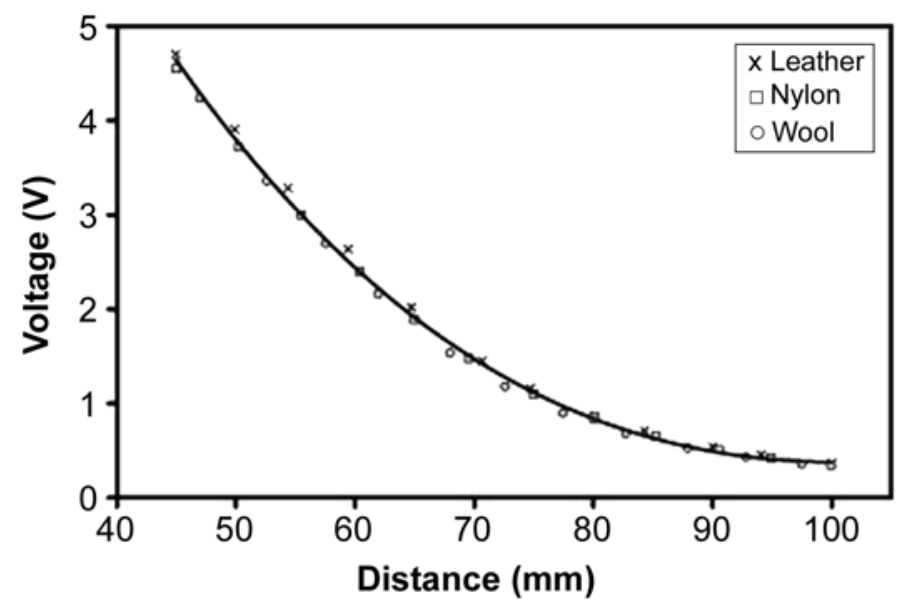

Figure 3.

Effects of different reflective material surfaces. Leather, nylon, and wool produced similar calibration test results. For clarity, data from only increasing portions of tests are shown.

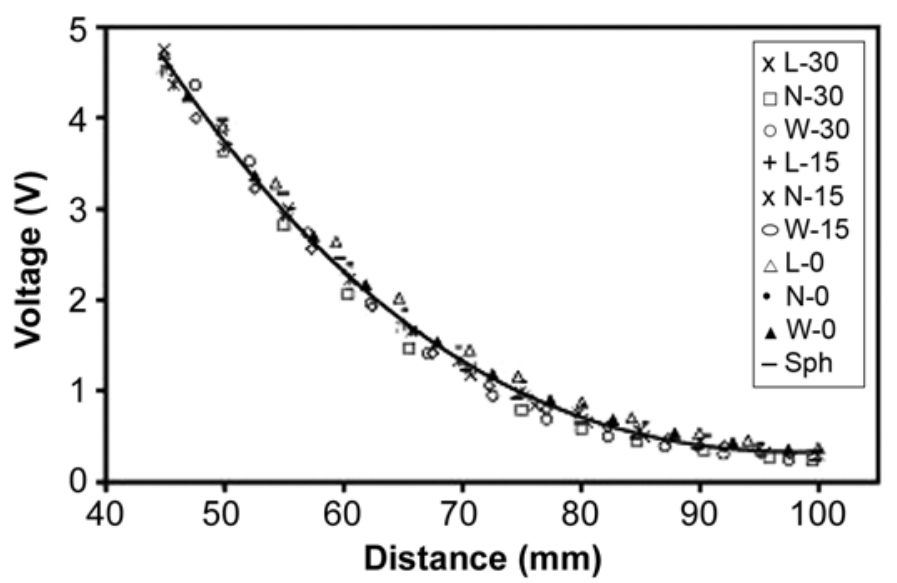

Figure 4.

Effects of different tilt angles and surface curvature. Leather (L), nylon $(\mathrm{N})$, and wool $(\mathrm{W})$ tested at $30^{\circ}(-30), 15^{\circ}(-15)$, and $0^{\circ}(-0)$ with respect to reflective surface produced comparable results in calibration testing. A $38 \mathrm{~mm}$-diameter hemisphere-shaped surface (Sph) was also tested. For clarity, data from only increasing portions of tests are shown. the error was 1.21 percent FSO. However, a wet nylon sock introduced a 3.08 percent FSO error to the measurement. We considered the third-order polynomial curve shown in Figure 4 for different angles, curvatures, and materials the calibration curve for the device. Using this curve in Figure 5 allows us to clearly see the influence of wetness, a source of error.

From dynamic testing, we detected no differences in gain or phase between the sensor and the LVDT over an input signal bandwidth of 0 to $60 \mathrm{~Hz}$. Thus, we considered the dynamic response of the sensor acceptable for this application. When considering all errors together (different reflective surfaces, peak-to-peak signal noise, drift, nonlinearity, different surface tilt angles, surface curvature, and wetness [simulating sweating]), we found that the RMS error for each material (leather, nylon, wool) was less than 1.95 percent FSO.

Results from testing on a transtibial amputee subject showed a distinct stance and swing phase pattern for each step (Figure 6). Approximately $0.2 \mathrm{~s}$ into stance phase, after a downward displacement of approximately $40 \mathrm{~mm}$, the limb position was relatively constant. Then at toe-off, the residual limb moved proximally in the socket and the prosthesis was lifted off the ground. During early swing phase, the limb immediately displaced proximally $\sim 30 \mathrm{~mm}$ and then another $\sim 10 \mathrm{~mm}$ within the next $0.2 \mathrm{~s}$ as the knee was pulled forward. Part of this $10 \mathrm{~mm}$ midswing phase displacement could have been due to limb motion within

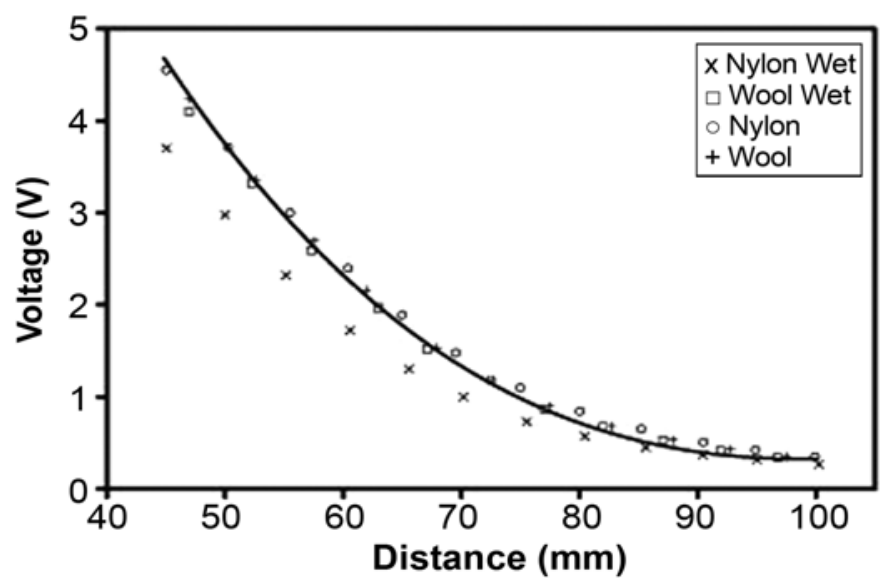

Figure 5.

Effects of wet surface. Wet nylon sock had more effect on calibration results than did wet wool sock. For clarity, data from only increasing portions of tests are shown. Polynomial curve shown is from Figure 4, calibration curve for different tilt angles and curvatures, which we considered calibration curve for device. 


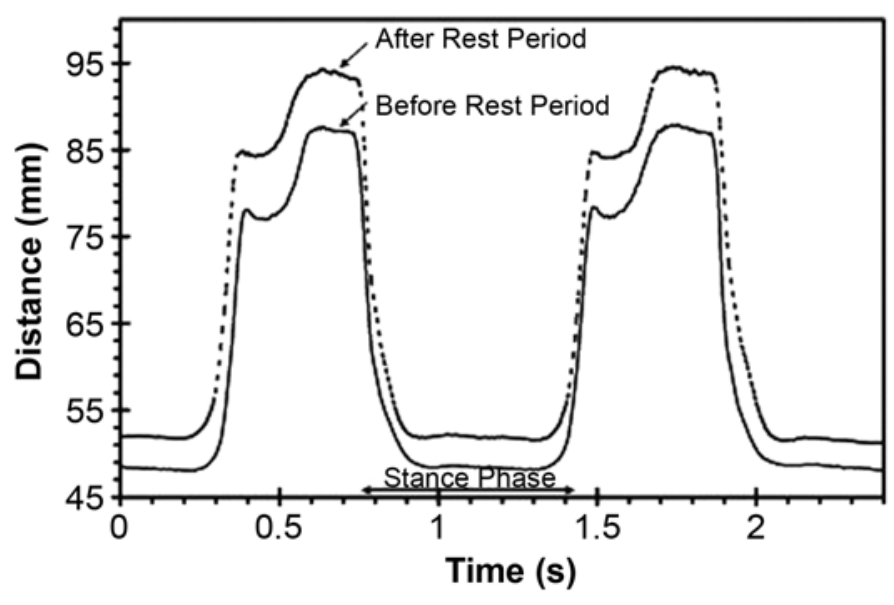

Figure 6.

Example results from testing of transtibial amputee subject showing stance and swing phase pattern. Position versus time for trials before and after 5 min rest period.

the socket. The region on the distal residual limb from where the sensor was sampled may have shifted during swing phase because of the anterior-posterior or mediallateral movement of the nonflat distal limb in the socket. However, we believed that the measurement represented the distal limb location. Minimal displacement occurred during late swing phase. After heel contact was reached and the ensuing stance phase started, the limb again displaced approximately $40 \mathrm{~mm}$ back down into the socket.

Differences resulted among the three test conditions. The total pistoning (most proximal position minus most distal position) while the subject was wearing the supracondylar strap was significantly less than when he was not wearing the supracondylar strap $(p=0.000)$. However, the magnitude of the mean difference was relatively small, just $0.8 \mathrm{~mm}$.

A position difference was seen for trials conducted without the supracondylar strap before versus after the rest period. Before the rest period, the average most proximal position within a step was $87.9 \pm 0.2$ standard deviation $\mathrm{mm}$ and the most distal position was $48.1 \pm 0.2 \mathrm{~mm}$. After the rest period, the positions were $94.5 \pm 0.4 \mathrm{~mm}$ and $51.0 \pm$ $0.4 \mathrm{~mm}$, respectively. Thus, the most proximal position of the residual limb in the socket moved proximally $\sim 6.6 \mathrm{~mm}$, and the most distal position moved proximally approximately $2.9 \mathrm{~mm}$. The average proximal displacement was thus $4.8 \mathrm{~mm}$. These results are consistent with the subject's comment that he felt like he was pulling out of the socket more in the trials after the rest period. A significant $(p=$ 0.000 ) total pistoning difference (most proximal position minus most distal position) also occurred. Before the rest period, pistoning was $39.8 \pm 0.2 \mathrm{~mm}$, and after the rest period, it was $43.5 \pm 0.3 \mathrm{~mm}$, an increase of $3.7 \mathrm{~mm}$. The average pistoning was $41.7 \pm 0.3 \mathrm{~mm}$. No sweat was evident on the prosthetic sock after data were collected.

\section{DISCUSSION}

A simple device for measuring the position of the distal residual limb in the prosthetic socket is of potential value in evaluating clinical features of interest that may affect position and pistoning. Insight into how different suspension systems during different activities affect pistoning or how different limb fluid control treatments affect proximal-distal limb position in the socket is possible with this device. A prosthetist could use the sensor clinically to measure the amount of pistoning that feels optimal to a patient and then use that information later during refitting.

Enhancements to the design described here would be needed for the sensor to be regularly used in a clinical setting. A limitation of the device is that a cable extends from the sensor to the display (computer data acquisition system). However, radio-frequency telemetry systems are now available (e.g., MIE Medical Research Ltd, Leeds, United Kingdom) that one could use to overcome this problem. Another issue is that a line of sight must exist between the sensing element and the distal limb (or sock). This limitation was acceptable in our subject trials because we used a patellar-tendon-bearing socket with a one-sock fit and a plastezote end pad. A small hole through the distal socket surface and the plastezote was the only socket modification we needed to install the device. A suspension liner with a pin-lock system, however, would not be usable with this device in its current form because no line of sight through the pin would be possible. Conceptually, one could position a smaller sensor (2 mm diameter, Balluff, Inc) within the locking pin to overcome this problem.

The length addition to the prosthesis with the sensor in place was $8.9 \mathrm{~mm}$. This addition was necessary so that we could include a frame that supported the rack and pinion. In clinical use, however, the distance between the distal socket and limb could be designed to be within the operating range of the sensor, the $60.0 \mathrm{~mm}$ window. Then the rack and pinion would be unnecessary and the height addition introduced by adding the sensor could be reduced to a few millimeters or less. The hole in the socket could be eliminated altogether, and the sensor mounted within a holder on the inside distal socket surface. 
The sensor output was nonlinearly related to the distance from the object, as is typical for electro-optical displacement transducers [10]. The nonlinearity was not a complication in data processing because it was not timedependent (i.e., no hysteresis). We accommodated for the nonlinearity in data processing using the calculated relationship between voltage and distance. Since the curve flattened at very high distances, the 95 to $100 \mathrm{~mm}$ region in Figures 3 to 5, absolute error was higher in this range than at lower distances.

The measurement was relatively insensitive to material (leather, nylon, wool), surface tilt angle (up to $30^{\circ}$ ), and surface curvature (38 $\mathrm{mm}$ diameter sphere), because we used a diffuse sensor, as opposed to a throughput sensor. Because much scatter occurred at the surface when we used the diffuse sensor, the reflected light intensity was not very sensitive to the incident angle. The feature that most substantially affected the measurement was the surface wetness, particularly for nylon. The water substantially changed the reflective characteristics of the surface, thus one should exercise caution when using the sensor with a nylon sock saturated with sweat, because this will increase error. We used a dry-surface calibration curve because, in our research, we have found that within a data acquisition session subjects rarely perspire enough distally to sweat all the way through the sock and make the outside of the sock wet. If such extensive sweating did occur, then one could use a calibration curve for wet nylon to reduce error.

The amount of pistoning measured for this subject, which averaged $41.7 \mathrm{~mm}$, is consistent with previous findings [1-9]. The additional mass added to the limb from the distal limb position instrumentation, i.e., sensor and frame, was not likely an important factor influencing pistoning. It was only $70 \mathrm{~g}$, approximately 3 percent of the mass of the prosthesis. Differences between the instrumented prosthesis and the subject's normal prosthesis should not have appreciably affected the comparisons here (with and without the supracondylar strap, before and after the rest period) because the same prosthesis was used in all tests.

Interestingly, the increase in pistoning without the strap versus with the strap was only $0.8 \mathrm{~mm}$. This result suggests that the supracondylar strap had little effect for this subject as he walked on a level surface. The neoprene sleeve provided enough support. The strap, however, could affect pistoning in other activities, such as stair-climbing. A topic of future interest is evaluating suspension systems for different activities.

The difference in limb-socket position before and after the rest period may reflect an increase in limb volume. Since this subject was diabetic, his residual limb would have been expected to swell quickly after he stopped walking. In a different study, limb volume after walking was shown to increase on most subjects at least 2 percent within a 5 min interval [13]. An enlarged limb would not be expected to displace as deeply into the socket during stance phase, which is consistent with the distal limb position data recorded here.

Our next step in this research will be to assess changes in distal limb position over time as the residual limb matures, treatments for residual limb volume change, and different suspension systems for different activities. We are also interested in simultaneously collecting interface stress data with limb position data to investigate a correlation between interface stress change and limb position change. With insight into the meaning of the data, the potential use of the sensor in clinical fitting and practice should emerge.

\section{CONCLUSIONS}

We developed a simple noncontact device to measure the position of the residual limb relative to the distal socket. The sensor and frame unit was $70 \mathrm{~g}$ in mass and added $8.9 \mathrm{~mm}$ to the socket length. The sensor had a range of $60.0 \mathrm{~mm}$ and an RMS error of less than 1.95 percent FSO. When used in a preliminary study on a transtibial amputee subject, the device measured an average of $41.7 \mathrm{~mm}$ of pistoning during walking. After a 5 min rest period, the position of the limb in the socket was $\sim 4.8 \mathrm{~mm}$ more proximal, possibly reflecting a limb volume increase as a result of the rest period.

\section{ACKNOWLEDGMENTS}

This material was based on work supported by the National Institutes of Health, National Institute of Biomedical Imaging and Bioengineering, grant R01EB4329.

The authors have declared that no competing interests exist. 


\section{REFERENCES}

1. Newton RL, Morgan D, Schreiber MH. Radiological evaluation of prosthetic fit in below-the-knee amputees. Skeletal Radiol. 1988;17(14):276-80. [PMID: 3212490]

2. Erikson U, Lemperg R. Roentgenological study of movements of the amputation stump within the prosthetic socket in below-knee amputees fitted with a PTB prosthesis. Acta Orthop Scand. 1969;40(4):520-29. [PMID: 5372254$]$

3. Grevsten S, Erikson U. A roentgenological study of the stump-socket contact and skeletal displacement in the PTBSuction Prosthesis. Ups J Med Sci. 1975;80(1):49-57. [PMID: 1145905]

4. Lilja M, Johansson T, Oberg T. Movement of the tibial end in a PTB prosthesis socket: A sagittal X-ray study of the PTB prosthesis. Prosthet Orthot Int. 1993;17(1):21-26. [PMID: 8337097]

5. Narita H, Yokogushi K, Shii S, Kakizawa M, Nosaka T. Suspension effect and dynamic evaluation of the total surface bearing (TSB) trans-tibial prosthesis: A comparison with the pateallar tendon bearing (PTB) trans-tibial prosthesis. Prosthet Orthot Int. 1997;21(3):175-78. [PMID: 9453088]

6. Kahle JT. A case study using fluoroscope to determine the vital elements of transfemoral interface design. J Prosthet Orthot. 2002;14(3):121-26.

7. Soderberg B. Roentgen stereophotogrammetric analysis of motion between the bone and the socket in a transtibial amputation prosthesis: A case study. J Prosthet Orthot. 2003; 15(3):95-99.

8. Murray KD, Convery P. The calibration of ultrasound transducers used to monitor motion of the residual femur within a trans-femoral socket during gait. Prosthet Orthot Int. 2000;24(1):55-62. [PMID: 10855439]

9. Convery P, Murray KD. Ultrasound study of the motion of the residual femur within a trans-femoral socket during gait. Prosthet Orthot Int. 2000;24(3):226-32. [PMID: 11195358]

10. Norton HN. Sensor and analyzer handbook. Englewood Cliffs (NJ): Prentice-Hall, Inc; 1982.

11. Berme N, Lawes P, Solomonidis S, Paul JP. A shorter pylon transducer for measurement of prosthetic forces and moments during amputee gait. Eng Med. 1976;4(4):6-8.

12. Sanders JE, Zachariah SG, Jacobsen AK, Fergason JR. Changes in interface pressures and shear stresses over time on trans-tibial amputee subjects ambulating with prosthetic limbs: Comparison of diurnal and six-month differences. J Biomech. 2005;38(8):1566-73. [PMID: 15958212]

13. Zachariah SG, Saxena R, Fergason JR, Sanders JE. Shape and volume change in the transtibial residuum over the short term: Preliminary investigation of six subjects. J Rehabil Res Dev. 2004;41(5):683-94. [PMID: 15558398]

14. Sanders JE, Daly CH, Burgess EM. Clinical measurement of normal and shear stresses on a trans-tibial stump: Characteristics of wave-form shapes during walking. Prosthet Orthot Int. 1993;17(1):38-48. [PMID: 8337099]

15. Mitchell SB, Sanders JE. An accurate inexpensive system for the assessment of walking speed. J Prosthet Orthot. 2000;12(4):117-19.

Submitted for publication November 17, 2004. Accepted in revised form February 13, 2006. 